\title{
ReCOMMANDATION No 3
}

Le Colleque international sur les problèmes biogéographiques de l'Upemba, réuni du 5 au 9 février 1963, à Elisabethville a adopté trois recommandations dont la $3 \mathrm{me}$ intéresse spécialement les hydrobioloques.

CONSIDERANT le grand intéret scientifique et pratique de l'étude de la productivité biologique des eaux, dans les pays ou la pèche fournit une part importante des ressources en protéines de la population,

Condiserant que les lacs peu profonds et marécageux sont des milieux aquatiques très particuliers en Afrique tropicale, et qu'ils sont les plus productifs du point de vue du rendement des pèches,

Recommande l'étude comparée de la bioproductivité des lacs de ce type du bassin du haut Congo (Lualaba et Luapula): lacs Bangweolo, Moero et Upemba,

INVITE les Institutions internationales et, en particulier, l'Organisation du Programme Biologique International, à accorder un appui effectif aux projets visant à une telle étude.

Président du colloque: KaY CURRY-LINDAHL, Stockholm. 